\title{
Assessing the upper motor neuron in amyotrophic lateral sclerosis using the triple stimulation technique: A multicenter prospective study
}

\author{
Aude-Marie Grapperon ${ }^{\mathrm{a}}$, Annie Verschueren ${ }^{\mathrm{a}}$, Elisabeth Jouve ${ }^{\mathrm{b}}$, Régine Morizot-Koutlidis ${ }^{\mathrm{c}}$, \\ Timothée Lenglet $^{c}$, Pierre-François Pradat ${ }^{d}$, François Salachas ${ }^{d}$, Emilien Bernard ${ }^{\mathrm{e}}$, Stéphanie Delstanche ${ }^{\mathrm{f}}$, \\ Alain Maertens de Noordhout ${ }^{\mathrm{f}}$, Nathalie Guy ${ }^{g}$, Véronique Danel ${ }^{\mathrm{h}}$, Arnaud Delval ${ }^{\mathrm{i}}$, Emilien Delmont ${ }^{\mathrm{a}}$, \\ Anne-Sophie Rolland ${ }^{\mathrm{h}}$, PULSE Study Group ${ }^{\mathrm{j}}$, Laurent Jomir ${ }^{\mathrm{e}}$, David Devos ${ }^{\mathrm{h}}$, François Wang ${ }^{\mathrm{k}}$, \\ Shahram Attarian ${ }^{\mathrm{a}, *}$
}

\footnotetext{
${ }^{a}$ APHM, Timone University Hospital, Referral Center for Neuromuscular Diseases and ALS, ERN Euro-NMD Center, Marseille, France

${ }^{\mathrm{b}}$ Aix Marseille Univ, APHM, INSERM, Inst Neurosci Syst, Department of Clinical Pharmacology and Pharmacovigilance, CIC-CPCET, Marseille, France

${ }^{\mathrm{c}}$ Department of Neurophysiology, APHP, Hôpital Pitié-Salpêtrière, Paris, France

${ }^{\mathrm{d}}$ Referral Center for ALS, APHP, Hôpital Pitié-Salpêtrière, Paris, France

e Service des pathologies neuromusculaires et du motoneurone, Hospices Civils de Lyon, France

${ }^{\mathrm{f}}$ University of Liège, Centre Hospitalier Régional de la Citadelle, Department of Neurology, Liège, Belgium

${ }^{g}$ CRC SLA et maladie du neurone moteur, U1107-neurodol-UCA, CHU de Clermont-Ferrand, France

${ }^{\mathrm{h}}$ University of Lille, Expert center for ALS, CHU-Lille, Lille Neuroscience \& Cognition, INSERM, UMR-S1172, LICEND, ACT4ALS-MND network, France

${ }^{i}$ Department of Clinical Neurophysiology, CHU-Lille, U1172 Lille Neuroscience E Cognition, University of Lille, France

j PULSE Study Group, France

${ }^{\mathrm{k}} \mathrm{CHU}$ de Liège, Department of Clinical Neurophysiology, Liège, Belgium
}

\section{A R T I C L E I N F O}

\section{Article history:}

Accepted 7 August 2021

Available online 12 August 2021

\section{Keywords:}

Amyotrophic lateral sclerosis

Upper motor neuron

Transcranial magnetic stimulation

Triple stimulation technique

\section{H I G H L I G H T S}

- - Triple stimulation technique can be used in routine clinical practice and in multicenter studies in ALS patients.

- - Assessment of corticospinal dysfunction at the first visit of the patient was improved by the use of triple stimulation technique compared to conventional TMS.

- - Triple stimulation technique results are correlated with the clinical upper motor neuron score and ALSFRS-R.

\begin{abstract}
A B S T R A C T
Objective: To evaluate the relevance of transcranial magnetic stimulation (TMS) using triple stimulation technique (TST) to assess corticospinal function in amyotrophic lateral sclerosis (ALS) in a large-scale multicenter study.

Methods: Six ALS centers performed TST and conventional TMS in upper limbs in 98 ALS patients during their first visit to the center. Clinical evaluation of patients included the revised ALS Functional Rating Scale (ALSFRS-R) and upper motor neuron (UMN) score.

Results: TST amplitude ratio was decreased in $62 \%$ of patients whereas conventional TMS amplitude ratio was decreased in $25 \%$ of patients and central motor conduction time was increased in $16 \%$ of patients. TST amplitude ratio was correlated with ALSFRS-R and UMN score. TST amplitude ratio results were not different between the centers.

Conclusions: TST is a TMS technique applicable in daily clinical practice in ALS centers for the detection of UMN dysfunction, more sensitive than conventional TMS and related to the clinical condition of the patients. Significance: This multicenter study shows that TST can be a routine clinical tool to evaluate UMN dysfunction at the diagnostic assessment of ALS patients.
\end{abstract}

(c) 2021 International Federation of Clinical Neurophysiology. Published by Elsevier B.V. All rights reserved.

\footnotetext{
* Corresponding author at: Service des Maladies Neuromusculaires et de la SLA, Hôpital de la Timone, 264 rue Saint Pierre, 13005 Marseille, France.

E-mail address: sattarian@ap-hm.fr (S. Attarian).
} 


\section{Introduction}

Among the major requirements to develop effective neuroprotective treatment for amyotrophic lateral sclerosis (ALS), the early therapeutic intervention is pivotal. This requires very sensitive diagnostic criteria. The diagnosis of ALS is based upon association of upper (UMN) and lower (LMN) motor neuron alteration. However, clinical UMN signs are sometimes difficult to assess in ALS patients, due either to the combination with LMN signs which may mask them or to their absence especially in the early stages of the disease. The development of objective UMN biomarkers is therefore necessary. Unlike electromyography (EMG) which has the same diagnostic significance as clinical LMN signs (de Carvalho et al., 2008), UMN signs are defined only by clinical examination in the revised El-Escorial diagnostic criteria (Brooks et al., 2000) as well as in the recent Gold Coast criteria (Shefner et al., 2020). Transcranial magnetic stimulation (TMS) techniques, though, have long shown their usefulness in assessing UMN dysfunction in ALS (de Carvalho et al., 2008; Huynh et al., 2018; Pouget et al., 2000; Ziemann and Eisen, 2004). However, to date, none of these techniques has shown enough diagnostic accuracy to be widely used in the diagnostic strategy of ALS patients. The results of TMS studies in ALS remain indeed variable, depending on the TMS technique and methodology used, the clinical heterogeneity and the disease stage of the patients. Hence, it is necessary to have a TMS technique applicable in clinical practice, evaluated in a large number of patients and by different teams and sensitive at the early stages of the disease.

Triple stimulation technique (TST) is a TMS technique developed by Magistris et al. providing a quantitative electrophysiological measure of the central motor conduction failure (Magistris et al., 1998). The desynchronization of the descending action potentials evoked by conventional TMS causes a variability in the size of the motor evoked potentials (MEPs) which therefore does not allow precise detection and quantification of corticospinal conduction failure. In healthy subjects as well as in patients, amplitudes of MEPs are indeed usually much smaller than those of motor responses to maximal peripheral nerve stimulation and show variation between normal subjects and from one stimulus to another. TST uses a collision technique to reduce the degree of desynchronization of MEPs and thus allows a more precise and reproducible quantification of the proportion of functional motor neurons emitting the descending corticomotoneuronal volley after activation by TMS. TST has been shown to be both sensitive and specific for the detection of UMN dysfunction in ALS patients (Attarian et al., 2007; Furtula et al., 2013; Grapperon et al., 2014; Kleine et al., 2010; Komissarow et al., 2004; Magistris et al., 1999; Rösler et al., 2000; Wang et al., 2019). However, TST is not yet widely used and requires validation with a large sample of patients at the diagnostic assessment through a multicenter prospective study.

\section{Methods}

\subsection{Study participants}

ALS patients were prospectively recruited in six ALS centers: Paris, Lyon, Lille, Clermont-Ferrand and Marseille, France and Liege, Belgium. The protocol was approved by the ethics committees. Written informed consent was obtained from all patients. Paris, Lille, Lyon, Marseille and Clermont-Ferrand centers included ALS patients in the French PULSE study (Study of Predictive Factors of Progression of Motor Neuron Disease, Protocol ID: 2013A00969-36; ClinicalTrials.gov: NCT02360891) coordinated by the University Hospital of Lille in partnership with the Association for the Research in Amyotrophic Lateral Sclerosis (ARSLA). In PULSE study, TST is part of the optional ancillary tests performed at the first patient visit for diagnostic assessment.

The diagnosis of the patients was made by the physicians of the ALS centers (V.D., E.B., L.J., F.W., S.D., A.M., F.S., P.F.P., T.L., N.G., A.M. G., E.D., A.V. and S.A.). Patients had to fulfil the revised El Escorial criteria for possible, probable-laboratory supported, probable or definite ALS (Brooks et al., 2000). Progressive muscular atrophy (PMA) patients were also included. PMA is the ALS subtype restricted to LMN (Ludolph et al., 2015) which was not included in the revised El Escorial criteria but in the recent Gold Coast criteria (Shefner et al., 2020). The diagnosis of PMA was made based on the presence of pure LMN clinical and EMG findings after ruling out other LMN diseases by extensive examinations, neuroimaging, biological and genetic tests. A standard electrodiagnostic evaluation was performed for all patients according to the Awaji criteria (de Carvalho et al., 2008) including full nerve conduction study testing of at least 8 motor and 6 sensory nerves and needle EMG of at least 4 muscles in lower limbs, 4 muscles in upper limbs, 2 muscles in thoracic region and 2 muscles in bulbar region. Patients were not included if they had other neurological conditions that could influence the results (e.g. stroke, cervical spondylotic myelopathy). All patients were followed up in the ALS centers allowing diagnosis confirmation.

\subsection{Clinical evaluation}

The clinical evaluation of the patients was performed on the day of the electrophysiological protocol and included disease duration, onset site, revised ALS functional rating scale (ALSFRS-R) (Cedarbaum et al., 1999), disease progression rate (i.e. [48ALSFRS-R]/disease duration in months) and an UMN score (Turner et al., 2004). The UMN score consisted of the number of pathologically brisk reflexes (biceps, triceps, supinator, knee, ankle, jaw jerk reflex, Hoffmann and Babinski signs), the maximal abnormal value was 15 .

\subsection{Electrophysiological protocol}

TMS techniques were performed by trained neurophysiologists (A.D., L.J., N.G., R.M.K., F.W., A.M.G. and S.A.). Annual meetings were organized for the participants to ensure the proper implementation of the protocol, share their practical experience and ensure the harmonization of the practice. TMS was performed by using a Magstim 200 stimulator (Magstim, Whitland, Wales) with a circular coil (9-cm diameter). Surface EMG signals were recorded with a bandpass filter of 3-10 $000 \mathrm{~Hz}$ using an EMG machine which was also used to perform electrical stimulation. Two types of EMG machine were used: Nicolet ${ }^{\circledR}$ Viking ${ }^{\circledR}$ EDX (Natus Medical Incorporated) in the centers of Marseille, Lyon, Lille and Clermont-Ferrand or Dantec ${ }^{\circledR}$ Keypoint ${ }^{\circledR}$ G4 EMG Workstation (Natus Medical Incorporated) in the centers of Paris and Liege. Compound muscle action potentials (CMAPs) of the right and left abductor digiti minimi (ADM) muscles were recorded with disposable disc electrodes in a tendon-belly configuration. TMS intensity was set at $130 \%$ of the resting motor threshold. TST was performed as described in previous studies (Attarian et al., 2015, 2007, 2005; Corazza et al., 2020; Deroide et al., 2007; Eusebio et al., 2007; Grapperon et al., 2019, 2014; Rico et al., 2009; Sevy et al., 2018) in accord with the practical guidelines for diagnostic TMS of the International Federation of Clinical Neurophysiology (Groppa et al., 2012). This technique consists first of the delivery of a magnetic stimulation on the motor cortex, followed by a supra-maximal electrical stimulation at the wrist on the ulnar nerve, so that the descending corticomotoneuronal volley collides with the antidromic action potentials. The collision takes place in the proximal portion of 
Table 1

Clinical characteristics of the 98 patients (mean value \pm SD).

\begin{tabular}{ll}
\hline Age (100\%) & $59 \pm 13$ yrs \\
Sex M/F (100\%) & $62 / 36(63 \% / 37 \%)$ \\
Disease duration (97\%) & $17 \pm 14$ months \\
Onset site (97\%) & Spinal: $77 \%$ (upper limb 40\%, lower limb 37\%) \\
& Bulbar: $22 \%$ \\
& Respiratory: $1 \%$ \\
Diagnostic criteria (97\%) & Definite ALS: $16 \%$ \\
& Probable ALS: $38 \%$ \\
& Probable-laboratory supported ALS: $19 \%$ \\
ALSFRS-R (/48) (100\%) & Possible ALS: $15 \%$ \\
Disease progression rate (/month) (99\%) & PMA: $12 \%$ \\
UMN score (/15) (54\%) & $40 \pm 6$ \\
\hline
\end{tabular}

The percentages of available data are noted in brackets in the first column. Abbreviations: ALS, amyotrophic lateral sclerosis; ALSFRS-R, revised ALS functional rating scale; PMA, progressive muscular atrophy; UMN, upper motor neuron

the nerve at the upper limb. A third and final stimulation is then delivered at the Erb point, allowing the acquisition of a highly synchronized response from the fibers in which the collision occurred. This response is compared to the response obtained after the control triple stimulation Erb point - wrist - Erb point to calculate TST amplitude ratio. TST amplitude ratio therefore reflects the number of functional neurons emitting the descending corticomotoneuronal volley.

Each center collected the following data for the left and right ADM muscles: 1) CMAP amplitudes elicited by peripheral electric stimulation of ulnar nerve at the wrist, 2) conventional TMS amplitude ratio (conventional TMS amplitude ratio = TMS MEP amplitude / CMAP amplitude), 3) central motor conduction time $(\mathrm{CMCT}=\mathrm{MEP}$ latency $-[$ minimal F-wave latency + CMAP latency - 1] /2), 4) TST amplitude ratio. TMS and TST were not performed if CMAP amplitude was $<1 \mathrm{mV}$.

Normal values of TST were developed in previous studies (Attarian et al., 2007; Magistris et al., 1999, 1998; Rösler et al., 2000). Control values were also obtained from 38 healthy subjects: mean value was $96.8 \%+/-3.87$, normal value using 2.5 SD limits was $87 \%$. No healthy subject had a TST amplitude ratio $<90 \%$, which is why this threshold of normal value was chosen. Laboratory standards established in the centers for the other parameters are as follows: abnormal conventional TMS amplitude ratio if $<33 \%$ and abnormal CMCT if $>10 \mathrm{~ms}$.

\subsection{Statistical analyses}

Statistical analyses were performed by using SAS software (version 9.4, SAS Institute, Cary, NC) and GraphPad Prism 5 (California, USA).

Given the heterogeneity and size of the sample, a Spearman rank test was used to evaluate associations between TST amplitude ratio and conventional TMS amplitude ratio as well as associations between TMS parameters (mean value between the right and left ADM TST amplitude ratio, conventional TMS amplitude ratio and $\mathrm{CMCT}$ ) and clinical data (ALSFRS-R and UMN score). The difference between the proportion of abnormal TST amplitude ratio and abnormal conventional TMS amplitude ratio on at least one side was analyzed by using the Fisher exact test.

The Kruskal-Wallis test and the Mann-Whitney-Wilcoxon test were used to determine differences in TMS parameters (TST amplitude ratio, conventional TMS amplitude ratio and $\mathrm{CMCT}$ ) between the centers. The difference of the percentage of patients with abnormal TST amplitude ratio, conventional TMS amplitude ratio and CMCT on at least one side between the centers was analyzed by using the Fisher exact test. We also verified that the patients of the centers were similar with regard to the distribution of clin- ical data by using the Fisher exact test for sex and onset site, the Kruskal-Wallis test for age, disease duration, disease progression rate, ALSFRS-R and UMN score. The Mann-Whitney test was used for pairwise comparisons with Tukey adjustment of p-values.

\section{Results}

\subsection{Study participants}

Ninety-eight patients were included in the study from January 13, 2016 to February 12, 2020. Fifty-two patients were included in the center of Marseille, 13 patients in the center of Paris, 12 patients in the center of Lyon, 10 patients in the center of Liege, 8 patients in the center of Lille and 3 patients in the center of Clermont-Ferrand.

Demographic and clinical characteristics of the patients are reported in Table 1.

\subsection{Conventional TMS and TST results}

Conventional TMS and TST results are given in Table 2.

TST amplitude ratio was abnormal on at least one side in 61/98 (62\%) patients while conventional TMS amplitude ratio was abnormal on at least one side in 21/84 (25\%) patients. TST amplitude ratio was significantly more often abnormal than conventional TMS amplitude ratio $(\mathrm{p}=0.001)$. TST amplitude ratio and conventional TMS amplitude ratio were correlated ( $\mathrm{rho}=0.68, \mathrm{p}<0.001$ for the right $\mathrm{ADM}$ and rho $=0.66, \mathrm{p}<0.001$ for the left $\mathrm{ADM}$ ) (Fig. 1).

TST and TMS results according to the diagnostic criteria of the patients are represented in Table 3. In the subgroup of PMA and possible ALS patients, 15/26 (58\%) patients had an abnormal TST amplitude ratio on at least one side while only $2 / 19$ (11\%) patients had an abnormal conventional TMS amplitude ratio on at least one side $(\mathrm{p}=0.002)$.

The rate of abnormal TST amplitude ratio in patients with clinical evidence of UMN in the limb tested was $88 \%$.

\subsection{Correlations between TMS and clinical data}

TST amplitude ratio was correlated with ALSFRS-R ( rho $=0.23$, $\mathrm{p}=0.03$ ) and with the UMN score (rho $=-0.31, \mathrm{p}=0.04$ ) (Fig. 2). Conventional TMS amplitude ratio was also correlated with the UMN score (rho $=-0.42, \mathrm{p}=0.01$ ) but not with ALSFRS-R (rho $=0.08, \mathrm{p}=0.48)$. CMCT was not correlated with ALSFRS-R ( $r$ o $=0.21, p=0.11$ ) nor with the UMN score ( rho $=0.14, p=0.27$ ). 
Table 2

TMS and TST results (mean value \pm SD)

\begin{tabular}{|c|c|c|c|c|}
\hline TMS parameters & CMAP (mV) & TST amplitude ratio (\%) & Conventional TMS amplitude ratio (\%) & CMCT (ms) \\
\hline Abnormal value & $<5.0$ & $<90$ & $<33$ & $>10$ \\
\hline Right ADM & $5.4 \pm 2.7(97 \%)$ & $75 \pm 34(91 \%)$ & $52 \pm 29(83 \%)$ & $8 \pm 4(64 \%)$ \\
\hline Left ADM & $5.3 \pm 2.7(97 \%)$ & $73 \pm 36(87 \%)$ & $50 \pm 30(82 \%)$ & $8 \pm 4(63 \%)$ \\
\hline$\%$ of patients with an abnormal result on at least one side & $57 \%$ & $62 \% * *$ & $25 \%$ & $16 \%$ \\
\hline
\end{tabular}

In brackets are noted the percentages of available data.

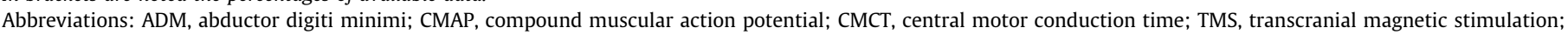
TST, triple stimulation technique.

** Comparison between TST amplitude ratio and conventional TMS amplitude ratio $(\mathrm{p}<0.01)$.

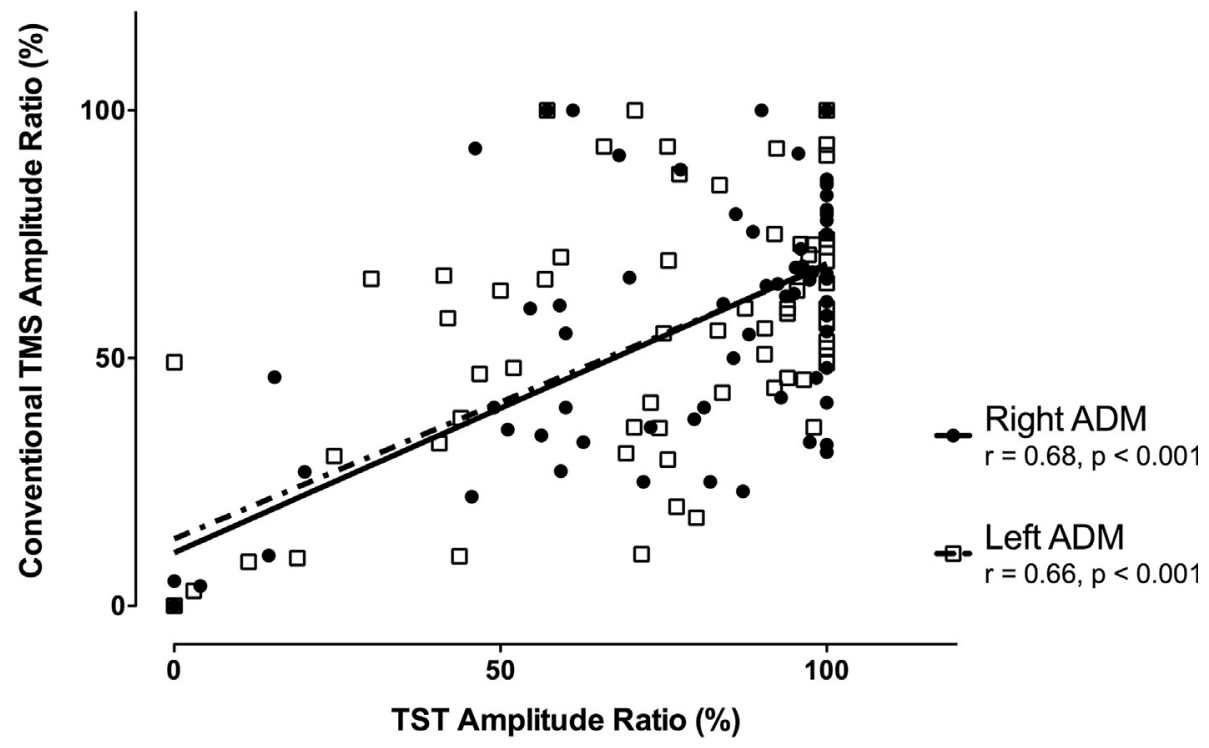

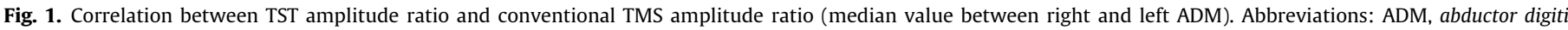
minimi; TMS, transcranial magnetic stimulation; TST, triple stimulation technique.

Table 3

TST and TMS results according to the diagnostic criteria of the patients.

\begin{tabular}{lll}
\hline $\begin{array}{l}\text { Diagnostic } \\
\text { criteria }\end{array}$ & $\begin{array}{l}\text { Number of patients with an } \\
\text { abnormal TST amplitude } \\
\text { ratio on at least one side / } \\
\text { total of patients }\end{array}$ & $\begin{array}{l}\text { Number of patients with an } \\
\text { abnormal conventional TMS } \\
\text { amplitude ratio on at least } \\
\text { one side / total of patients }\end{array}$ \\
\hline $\begin{array}{c}\text { Definite ALS } \\
(\mathrm{n}=15)\end{array}$ & $11 / 15(73.3 \%)$ & $3 / 13(23.1 \%)$ \\
$\begin{array}{c}\text { Probable ALS } \\
(\mathrm{n}=36)\end{array}$ & $24 / 36(66.7 \%)$ & $13 / 34(38.2 \%)$ \\
$\begin{array}{c}\text { Probable } \\
\text { laboratory } \\
\text { supported } \\
\text { ALS (n=18) }\end{array}$ & $11 / 18(61.1 \%)$ & $3 / 18(16.7 \%)$ \\
$\begin{array}{c}\text { Possible ALS } \\
(\mathrm{n}=14)\end{array}$ & $9 / 14(64.3 \%)$ & $1 / 8(12.5 \%)$ \\
PMA (n=12) & $6 / 12(50 \%)$ & $1 / 9(11.1 \%)$ \\
\hline
\end{tabular}

Abbreviations: ALS, amyotrophic lateral sclerosis; PMA, progressive muscular atrophy; TMS, transcranial magnetic stimulation; TST, triple stimulation technique.

\subsection{Comparison of TMS results among centers}

The TST results of the six centers are given in Table 4 and are represented in Fig. 3. Clermont-Ferrand center's data was not used for the comparative analysis between the centers due to the small number of patients included. TST amplitude ratio was not significantly different between the centers $(p=0.65$ for the right ADM, $\mathrm{p}=0.19$ for the left ADM). The other TMS parameters were not significantly different either $(p>0.05)$ except for the conventional TMS amplitude ratio of the left ADM (Kruskal-Wallis test $\mathrm{p}=0.04$, no significant pairwise comparison; $\mathrm{p}<0.05$ adjusted Tukey).

Regarding the clinical data of the patients, ALSFRS-R was significantly different between the centers (Kruskal-Wallis test, $\mathrm{p}=0.03$ ), ALSFRS-R was lower in Paris than in Lyon (MannWhitney test, $\mathrm{p}=0.01$ ) and was not significantly different between the other centers (Mann-Whitney test, $\mathrm{p}>0.05$ ). UMN score was significantly different between the centers (Kruskal-Wallis test, $\mathrm{p}=0.01$ ), UMN score was lower in Lyon than in Marseille (MannWhitney test, $\mathrm{p}=0.01$ ) and was not significantly different between the other centers (Mann-Whitney test, $\mathrm{p}>0.05$ ). The other clinical data including age, sex, onset site, disease duration and disease progression rate were not significantly different between the centers $(\mathrm{p}>0.05)$.

The percentage of patients with an abnormal TST amplitude ratio, conventional TMS amplitude ratio or CMCT on at least one side was not significantly different between the centers $(p>0.05)$.

\section{Discussion}

This multicenter study shows that TST can be performed by different centers in a large number of ALS patients and improves significantly the results obtained with conventional TMS techniques.

TMS abnormalities commonly found in ALS, prolonged CMCT and small or unobtainable MEPs, lack sensitivity and the results of the different studies are somewhat inconsistent (Chen et al., 2008). The variability in the results can be explained by the difference between TMS techniques and methods used and by the differ- 

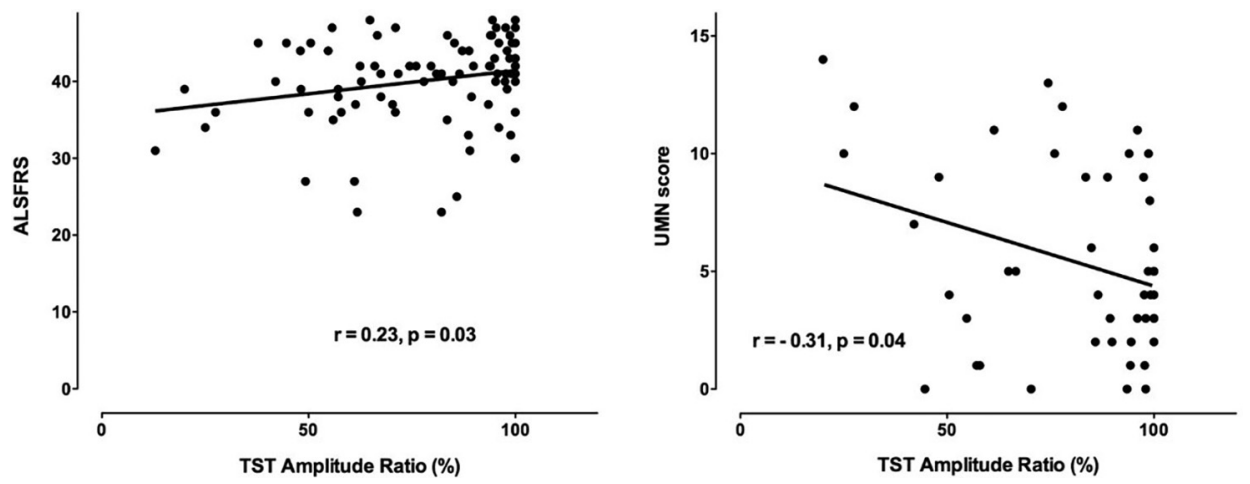

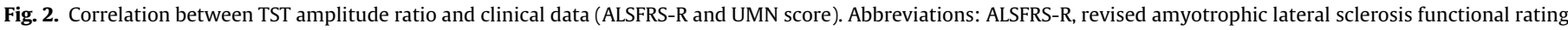
scale; TST, triple stimulation technique; UMN, upper motor neuron.

Table 4

TST results in the six centers (mean $\pm \mathrm{SD}$ ).

\begin{tabular}{lll}
\hline Center & $\begin{array}{l}\text { TST amplitude ratio right } \\
\text { ADM }(\%)\end{array}$ & $\begin{array}{l}\text { TST amplitude ratio left } \\
\text { ADM }(\%)\end{array}$ \\
\hline Paris $(\mathrm{n}=13)$ & $73 \pm 34$ & $66 \pm 38$ \\
Marseille $(\mathrm{n}=52)$ & $68 \pm 38$ & $63 \pm 37$ \\
Lyon $(\mathrm{n}=12)$ & $87 \pm 24$ & $83 \pm 26$ \\
Liège $(\mathrm{n}=10)$ & $85 \pm 19$ & $86 \pm 18$ \\
Lille $(\mathrm{n}=8)$ & $80 \pm 18$ & $79 \pm 18$ \\
Clermont-Ferrand & $92 \pm 14$ & $100 \pm 0$ \\
$\quad(\mathrm{n}=3)$ & \\
\hline
\end{tabular}

Abbreviations: ADM, abductor digiti minimi; TST, triple stimulation technique.

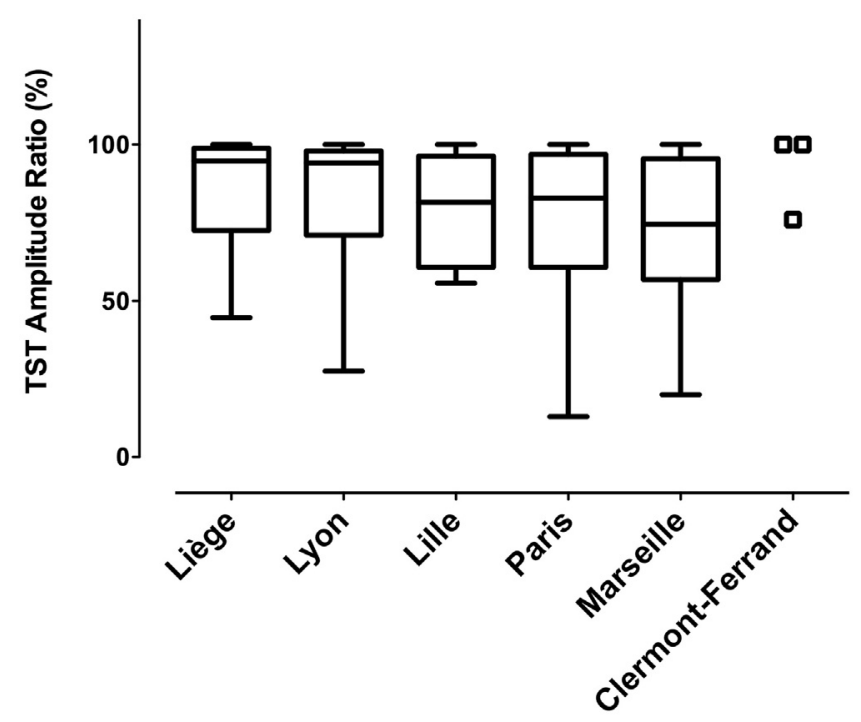

Fig. 3. TST amplitude ratio distribution in the 6 centers. Abbreviation: TST, triple stimulation technique.

ence in the clinical condition of the patients examined. Indeed, studies are often based on a small sample size of ALS patients while TMS results are influenced by the stage of the disease and the clinical presentation of the patients (UMN vs LMN-predominant or bulbar vs spinal forms for example). This study included a large number of patients who were examined at the time of their diagnostic assessment, which allowed to evaluate in real life the diagnostic interest of TST.

TST has been shown to be at least two times more sensitive than conventional TMS to detect corticospinal conduction deficit in ALS (Attarian et al., 2007; Bühler et al., 2001; Kleine et al., 2010;
Komissarow et al., 2004; Rösler et al., 2000), which has been confirmed by this study showing an abnormal amplitude ratio with TST in $62 \%$ of ALS patients against $25 \%$ with conventional TMS. Decreased conventional TMS amplitude ratio can be explained by the corticospinal conduction failure occurring in ALS due to UMN dysfunction. However, it is not a sensitive marker because MEP size is a variable parameter even in healthy subjects and, in ALS, the desynchronization of the descending volleys after TMS is often abnormally increased (Attarian et al., 2008, 2006; Awiszus and Feistner, 1993; Mills, 1995). TST synchronizes the response of the motor neurons driven to discharge by TMS, thereby avoiding phase cancellation that accompanies the desynchronization of the biphasic motor unit potentials and eliminates repetitive discharges from the measured response. Unlike conventional TMS amplitude ratio measurement, TST therefore allows a precise quantification estimation of corticospinal conduction failure caused by UMN dysfunction which probably occurs early in the disease. Another conventional TMS parameter that is often abnormal in ALS is CMCT. CMCT measurement allows an estimate of the conduction time of corticospinal fibers between motor cortex and LMN. CMCT prolongation in ALS can be explained by the degeneration of the fastest conducting corticospinal fibers. CMCT measurement has also been shown useful to detect UMN dysfunction in ALS patients without clinically predominant UMN signs but may have low sensitivity in the early stages of the disease (Eisen et al., 1996; Floyd et al., 2009; Huynh et al., 2018; Kohara et al., 1996; Mills, 2003; Tokimura et al., 2020), which is confirmed by this study showing that CMCT is rarely abnormal at the time of the patient's initial diagnostic visit.

This study confirms that TST is an easy-to-perform and widely usable technique in clinical practice. The TST exam lasts about 20 minutes and is well tolerated by the majority of patients, although electrical stimulation at Erb's point is very often considered uncomfortable as in nerve conduction study. Good TST practice is easy to learn and perform well for neurophysiologists experienced in practicing TMS. A half-day training session is usually sufficient to learn TST practice for confirmed neurophysiologists.

This study focused on TST, one of the most accurate neurophysiological measure to detect corticospinal conduction deficits, but TST does not allow the study of cortical inhibition and facilitation. Other TMS techniques involving paired-pulse have studied cortical excitability in ALS (Vucic et al., 2013). Threshold-tracking TMS studies have thus shown that an absent or reduced short-interval intracortical inhibition is an early marker in ALS with high sensitivity and specificity (Menon et al., 2015; Vucic et al., 2018, 2011). However, these TMS techniques are not yet widely used in clinical practice. 
This multicenter study shows that TST can be used by different centers in clinical practice and has allowed this technique to be studied in a large number of patients. A limitation of this study is that the number of patients included in several institutions was small. Thus, the results of the analyses of institutional differences should be interpreted with caution and need to be confirmed in larger multicenter studies. However, despite this limitation, consistent results were observed between the different centers. Another limitation of the study is the absence of a control group. Normal values have been developed in previous studies (Attarian et al., 2007; Magistris et al., 1999, 1998; Rösler et al., 2000). The limit of normal TST value of $90 \%$ was established because in previous studies no healthy control had a TST amplitude ratio < 90\%. In addition, each of the centers had been trained by Michel Magistris and the harmonization of the technique was verified for this study by training sessions. Lastly, another limitation of the study is that TST was only performed in the upper limbs. Adding TST in the lower limbs could further improve sensitivity but is a more invasive technique. TST and TMS techniques are also limited in cases of important limb atrophy (CMAP $<1 \mathrm{mV}$ ).

Interestingly, TST amplitude ratio correlated with the clinical scores including the ALSFRS-R that is the gold standard measure of clinical incapacity in ALS and the UMN score. It is indeed important that an electrophysiological marker used in daily practice be correlated with the clinical incapacity of patients. These correlations were however low (ALSFRS-R, rho $=0.23$; UMN score, rho $=-0.31$ ). This may be explained by the fact that these clinical scores depend on both LMN and UMN involvement whereas TST explores UMN dysfunction. The presence of a significant correlation between UMN score and TST abnormalities shows that the corticospinal dysfunction measured by TST could have a clinical implication. UMN score is an easy-to-use score allowing rapid clinical assessment of UMN signs. This score was only available for 53/98 patients because it was included in the optional clinical data set.

In this study, half of the PMA patients had an abnormal TST amplitude ratio. The perspective is therefore to study a larger number of PMA patients since TMS techniques have the greatest diagnostic utility for these patients. These results are in line with post mortem studies capturing subclinical corticospinal tract degeneration in half of the cases with PMA (Ince et al., 2003). TST which allows detecting subclinical UMN involvement can thus be useful in improving the diagnosis of PMA which is often challenging. TST is also useful for patients in whom UMN signs are unclear, for example limited to brisk reflexes, since clinical UMN signs may be masked by LMN signs, or which appear later in the course of the disease. The sensitivity of ALS diagnostic criteria could be improved by the addition of TMS techniques and a dedicated study would be interesting to evaluate it. However, the main question of whether atypical ALS phenotypes such as PMA should be included in therapeutic trials remains unresolved as their response to treatments may be different from classical ALS.

In conclusion, this multicenter study shows that TST can be used in routine clinical practice in ALS patients and improves the assessment of corticospinal dysfunction compared to conventional TMS at the first evaluation of the patients.

\section{Declaration of Competing Interest}

The authors declare that they have no known competing financial interests or personal relationships that could have appeared to influence the work reported in this paper.

\section{Acknowledgements}

This work was supported by the Association for the Research in Amyotrophic Lateral Sclerosis (ARSLA).

\section{Appendix A. Supplementary data}

Supplementary data to this article can be found online at https://doi.org/10.1016/j.clinph.2021.08.003.

\section{References}

Attarian S, Azulay J-P, Verschueren A, Pouget J. Magnetic stimulation using a triplestimulation technique in patients with multifocal neuropathy without conduction block. Muscle Nerve 2005;32(6):710-4. https://doi.org/10.1002/ (ISSN)1097-459810.1002/mus.v32:610.1002/mus.20434

Attarian S, Franques J, Elisabeth J, Trébuchon A, Duclos Y, Wybrecht D, Verschueren A, Salort-Campana E, Pouget J. Triple-stimulation technique improves the diagnosis of chronic inflammatory demyelinating polyradiculoneuropathy. Muscle Nerve 2015;51(4):541-8. https://doi.org/10.1002/mus.24352.

Attarian S, Vedel J-P, Pouget J, Schmied A. Progression of cortical and spinal dysfunctions over time in amyotrophic lateral sclerosis. Muscle Nerve 2008;37 (3):364-75. https://doi.org/10.1002/mus.v37:310.1002/mus.20942.

Attarian S, Vedel J-P, Pouget J, Schmied A. Cortical versus spinal dysfunction in amyotrophic lateral sclerosis. Muscle Nerve 2006;33(5):677-90. https://doi. org/10.1002/(ISSN)1097-459810.1002/mus.v33:510.1002/mus.20519.

Attarian S, Verschueren A, Pouget J. Magnetic stimulation including the triplestimulation technique in amyotrophic lateral sclerosis. Muscle Nerve 2007;36 (1):55-61. $\quad$ https://doi.org/10.1002/(ISSN)1097-459810.1002/mus. v36:110.1002/mus.20789.

Awiszus F, Feistner H. Abnormal EPSPs evoked by magnetic brain stimulation in hand muscle motoneurons of patients with amyotrophic lateral sclerosis. Electroencephalogr Clin Neurophysiol 1993;89(6):408-14. https://doi.org/ 10.1016/0168-5597(93)90114-5.

Brooks BR, Miller RG, Swash M, Munsat TL. World Federation of Neurology Research Group on Motor Neuron Diseases. El Escorial revisited: revised criteria for the diagnosis of amyotrophic lateral sclerosis. Amyotroph Lateral Scler Other Motor Neuron Disord 2000;1(5):293-9.

Bühler R, Magistris MR, Truffert A, Hess CW, Rösler KM. The triple stimulation technique to study central motor conduction to the lower limbs. Clin Neurophysiol 2001;112(5):938-49. https://doi.org/10.1016/S1388-2457(01) 00506-5.

de Carvalho M, Dengler R, Eisen A, England JD, Kaji R, Kimura J, Mills K, Mitsumoto $\mathrm{H}$, Nodera H, Shefner J, Swash M. Electrodiagnostic criteria for diagnosis of ALS. Clin Neurophysiol 2008;119(3):497-503. https://doi.org/10.1016/ i.clinph.2007.09.143.

Cedarbaum JM, Stambler N, Malta E, Fuller C, Hilt D, Thurmond B, Nakanishi A. The ALSFRS-R: a revised ALS functional rating scale that incorporates assessments of respiratory function. BDNF ALS Study Group (Phase III). J Neurol Sci 1999;169 (1-2):13-21.

Chen R, Cros D, Curra A, Di Lazzaro V, Lefaucheur J-P, Magistris MR, Mills K, Rösler $\mathrm{KM}$, Triggs WJ, Ugawa Y, Ziemann U. The clinical diagnostic utility of transcranial magnetic stimulation: report of an IFCN committee. Clin Neurophysiol j.clinph.2007.10.014.

Corazza G, Le Corroller T, Grapperon AM, Salort-Campana E, Verschueren A, Attarian S, Delmont E. Comparison of MRI and motor evoked potential with triple stimulation technique for the detection of brachial plexus abnormalities in multifocal motor neuropathy. Muscle Nerve 2020;61(3):325-9. https://doi.org/ 10.1002/mus.v61.310.1002/mus.26773.

Deroide N, Uzenot D, Verschueren A, Azulay JP, Pouget J, Attarian S. Triplestimulation technique in multifocal neuropathy with conduction block. Muscle Nerve 2007;35(5):632-6. https://doi.org/10.1002/(ISSN)1097459810.1002/mus.v35:510.1002/mus.20742.

Eisen A, Entezari-Taher M, Stewart H. Cortical projections to spinal motoneurons: changes with aging and amyotrophic lateral sclerosis. Neurology 1996;46:1396-404. https://doi.org/10.1212/wnl.46.5.1396.

Eusebio A, Azulay J-P, Witjas T, Rico A, Attarian S. Assessment of cortico-spinal tract impairment in multiple system atrophy using transcranial magnetic stimulation. Clin Neurophysiol 2007;118(4):815-23. https://doi.org/10.1016/ j.clinph.2007.01.004.

Floyd AG, Yu QP, Piboolnurak P, Tang MX, Fang Y, Smith WA, Yim J, Rowland LP, Mitsumoto H, Pullman SL. Transcranial magnetic stimulation in ALS: utility of central motor conduction tests. Neurology 2009;72(6):498-504. https://doi. org/10.1212/01.wnl.0000341933.97883.a4

Furtula J, Johnsen B, Frandsen J, Rodell A, Christensen PB, Pugdahl K, FuglsangFrederiksen A. Upper motor neuron involvement in amyotrophic lateral sclerosis evaluated by triple stimulation technique and diffusion tensor MRI. J Neurol 2013;260(6):1535-44. https://doi.org/10.1007/s00415-012-6824-8.

Grapperon A-M, Ridley B, Verschueren A, Maarouf A, Confort-Gouny S, Fortanier E, Schad L, Guye M, Ranjeva J-P, Attarian S, Zaaraoui W. Quantitative Brain Sodium MRI Depicts Corticospinal Impairment in Amyotrophic Lateral Sclerosis. Radiology 2019;292(2):422-8. https://doi.org/10.1148/radiol.2019182276.

Grapperon A-M, Verschueren A, Duclos Y, Confort-Gouny S, Soulier E, Loundou AD, Guye M, Cozzone PJ, Pouget J, Ranjeva J-P, Attarian S. Association between structural and functional corticospinal involvement in amyotrophic lateral sclerosis assessed by diffusion tensor MRI and triple stimulation technique. Muscle Nerve 2014;49(4):551-7. https://doi.org/10.1002/mus.23957. 
Groppa S, Oliviero A, Eisen A, Quartarone A, Cohen LG, Mall V, Kaelin-Lang A, Mima T, Rossi S, Thickbroom GW, Rossini PM, Ziemann U, Valls-Solé J, Siebner HR. A practical guide to diagnostic transcranial magnetic stimulation: report of an IFCN committee. Clin Neurophysiol 2012;123(5):858-82. https://doi.org/ 10.1016/i.clinph.2012.01.010.

Huynh W, Dharmadasa T, Vucic S, Kiernan MC. Functional Biomarkers for Amyotrophic Lateral Sclerosis. Front Neurol 2018;9:1141. https://doi.org/ 10.3389/fneur.2018.01141.

Ince PG, Evans J, Knopp M, Forster G, Hamdalla HHM, Wharton SB, Shaw PJ. Corticospinal tract degeneration in the progressive muscular atrophy variant of ALS. Neurology 2003;60(8):1252-8. https://doi.org/10.1212/01. WNL.0000058901.75728.4E

Kleine BU, Schelhaas HJ, van Elswijk G, de Rijk MC, Stegeman DF, Zwarts MJ. Prospective, blind study of the triple stimulation technique in the diagnosis of ALS. Amyotroph Lateral Scler 2010;11(1-2):67-75. https://doi.org/10.3109/ 17482960903171128.

Kohara N, Kaji R, Kojima Y, Mills KR, Fujii H, Hamano T, Kimura J, Takamatsu N, Uchiyama T. Abnormal excitability of the corticospinal pathway in patients with amyotrophic lateral sclerosis: a single motor unit study using transcranial magnetic stimulation. Electroencephalogr Clin Neurophysiol 1996;101 (1):32-41. https://doi.org/10.1016/0013-4694(95)00166-2.

Komissarow L, Rollnik JD, Bogdanova D, Krampfl K, Khabirov FA, Kossev A, et al. Triple stimulation technique (TST) in amyotrophic lateral sclerosis. Clin Neurophysiol 2004;115:356-60. https://doi.org/10.1016/i.clinph.2003.10.003.

Ludolph A, Drory V, Hardiman O, Nakano I, Ravits J, Robberecht W, Shefner J. A revision of the El Escorial criteria - 2015. Amyotroph Lateral Scler Frontotemporal Degener 2015;16(5-6):291-2. https://doi.org/10.3109/ 21678421.2015.1049183.

Magistris MR, Rösler KM, Truffert A, Landis T, Hess CW. A clinical study of motor evoked potentials using a triple stimulation technique. Brain 1999;122:265-79.

Magistris MR, Rösler KM, Truffert A, Myers JP. Transcranial stimulation excites virtually all motor neurons supplying the target muscle. A demonstration and a method improving the study of motor evoked potentials. Brain 1998;121:437-50. https://doi.org/10.1093/brain/121.3.437.

Menon P, Geevasinga N, Yiannikas C, Howells J, Kiernan MC, Vucic S. Sensitivity and specificity of threshold tracking transcranial magnetic stimulation for diagnosis of amyotrophic lateral sclerosis: a prospective study. Lancet Neurol 2015;14 (5):478-84. https://doi.org/10.1016/S1474-4422(15)00014-9.

Mills KR. The natural history of central motor abnormalities in amyotrophic lateral sclerosis. Brain 2003;126(11):2558-66. https://doi.org/10.1093/brain/awg260.

Mills KR. Motor neuron disease. Studies of the corticospinal excitation of single motor neurons by magnetic brain stimulation. Brain 1995;118:971-82.

Pouget J, Trefouret S, Attarian S. Transcranial magnetic stimulation (TMS): compared sensitivity of different motor response parameters in ALS.
Amyotroph Lateral Scler Other Motor Neuron Disord 2000;1(Suppl 2):S45-9. https://doi.org/10.1080/14660820052415817.

Rico A, Audoin B, Franques J, Eusebio A, Reuter F, Malikova I, Ali Cherif A, Pouget J, Pelletier J, Attarian S. Motor evoked potentials in clinically isolated syndrome suggestive of multiple sclerosis. Mult Scler 2009;15(3):355-62. https://doi.org/ $10.1177 / 1352458508099612$.

Rösler KM, Truffert A, Hess CW, Magistris MR. Quantification of upper motor neuron loss in amyotrophic lateral sclerosis. Clin Neurophysiol 2000;111(12):2208-18. https://doi.org/10.1016/S1388-2457(00)00481-8.

Sevy A, Grapperon A-M, Salort Campana E, Delmont E, Attarian S. Detection of proximal conduction blocks using a triple stimulation technique improves the early diagnosis of Guillain-Barré syndrome. Clin Neurophysiol 2018;129 (1):127-32. https://doi.org/10.1016/i.clinph.2017.10.035.

Shefner JM, Al-Chalabi A, Baker MR, Cui LY, de Carvalho M, Eisen A, Grosskreutz J, Hardiman O, Henderson R, Matamala JM, Mitsumoto H, Paulus W, Simon N Swash M, Talbot K, Turner MR, Ugawa Y, van den Berg LH, Verdugo R, Vucic S, Kaji R, Burke D, Kiernan MC. A proposal for new diagnostic criteria for ALS. Clin $\begin{array}{lll}\text { Neurophysiol } & \text { 2020;131(8):1975-8. } & \text { https://doi.org/10.1016 }\end{array}$ i.clinph.2020.04.005.

Tokimura R, Murakami T, Ugawa Y. Central motor conduction time reveals upper motor neuron involvement masked by lower motor neuron impairment in a significant portion of patients with amyotrophic lateral sclerosis. Clin Neurophysiol 2020;131(8):1896-901. https://doi.org/10.1016/ j.clinph.2020.05.021.

Turner MR, Cagnin A, Turkheimer FE, Miller CCJ, Shaw CE, Brooks DJ, Leigh PN, Banati RB. Evidence of widespread cerebral microglial activation in amyotrophic lateral sclerosis: an [11C](R)-PK11195 positron emission tomography study. Neurobiol Dis 2004;15(3):601-9. https://doi.org/10.1016/i. nbd.2003.12.012.

Vucic S, van den Bos M, Menon P, Howells J, Dharmadasa T, Kiernan MC. Utility of threshold tracking transcranial magnetic stimulation in ALS. Clin Neurophysiol Pract 2018;3:164-72. https://doi.org/10.1016/j.cnp.2018.10.002.

Vucic S, Cheah BC, Yiannikas C, Kiernan MC. Cortical excitability distinguishes ALS from mimic disorders. Clin Neurophysiol 2011;122(9):1860-6. https://doi.org/ 10.1016/i.clinph.2010.12.062.

Vucic S, Ziemann U, Eisen A, Hallett M, Kiernan MC. Transcranial magnetic stimulation and amyotrophic lateral sclerosis: pathophysiological insights. J Neurol Neurosurg Psychiatr 2013;84(10):1161-70. https://doi.org/10.1136/ innp-2012-304019.

Wang Y, Wang H, Cui LY. Triple Stimulation Technique in Amyotrophic Lateral Sclerosis. J Clin Neurophysiol 2019;36(2):87-92. https://doi.org/10.1097/ WNP.0000000000000520.

Ziemann U, Eisen A. TMS for ALS: why and why not. Clin Neurophysiol 2004;115 (6):1237-8. https://doi.org/10.1016/j.clinph.2004.02.008. 